\title{
COMUNICACIÓN ESTRATÉGICA: INDICADORES COMUNICACIONALES EN LA DIMENSIÓN INTERACCIONAL
}

\section{STRATEGIC COMMUNICATION: COMMUNICATIONAL INDICATORS ON INTERACTIONAL DIMENSION}

\section{Páginas: \\ Recibido: \\ 23 de octubre de 2014 \\ Aceptado}

Sandra Massoni

12 de mayo de 2015

El artículo se desprende de proyecto "Indicadores de comunicación en dimensiones múltiples", llevado a cabo entre

2011 y 2014 en la Maestría en Comunicación Estratégica de la Universidad Nacional de Rosario,

Argentina. de Argentina

Javier Spagnolo sandra.massonidafcpolit.unr.edu.ar

Catedratica de comunicación estratégica -categoría l-.

investigadora y docente de posgrados en Argentina y en

otros países. Entre sus publicaciones están La comunicación como herramienta estratégica (1990) y Metodologías de

la comunicación estratégica: del inventario al encuentro sociocultural (2013). Evaluadora CONICET y CONEAU. Dirige la Maestría en Comunicación Estratégica/Especialización en Comunicación Ambiental en la Universidad Nacional de Rosario

spagnolo.javier囚inta.gob.ar

Magister en Gestión de la Comunicación en las Organizaciones (Universidad Austral), Licenciado en Comunicación Social, egresado de la Facultad de Periodismo y Comunicación Social de la Universidad Nacional de La Plata. Es miembro de la Escuela de Comunicación Estratégica de Rosario, investigador en comunicación y profesional del Instituto Nacional de Tecnología Agropecuaria de Argentin

\section{Evelyn Gerson}

evelyn.s.gersonđagmail.com

Licenciada en Comunicación Social (UNR), investigadora, adscripta de grado y profesional de la Comunicación

Estratégica. Especialista en Responsabilidad Social Empresaria y Desarrollo Sostenible (UCEL). Trabaja de forma autónoma coordinando programas y proyectos educativos y culturales para ONGs y empresas privadas. Es miembro de la Escuela de Comunicación Estratégica de Rosario.
Resumen

Este artículo es un informe de avance de una investigación que propone indagar la dimensión comunicacional en organizaciones de distintos ámbitos, como un aporte al diseño y la implementación de las acciones comunicacial conversacional. El Proyecto "Indicadores comunicacionales en dimensiones múltiples" tiene como objetivo construir indicadores en cuatro dimensiones de la comunicación informativa, interaccional, ideológica y sociocultural. Este artículo presenta procesos y resultados obtenidos en la construcción, elección, relevamiento y registro de indicadores en la dimensión interaccional que, en el entorno de una plataforma digital, permiten un monitoreo continuo de actividades, proceun monitoreo continuo de actividades, proce-
sos y productos en el marco de proyectos de comunicación estratégica en programas, organizaciones y empresas.

Palabras clave Comunicación estratégica, metodología, dimensión interaccional, indicadores comunicacionales.

\section{Abstract}

This article is a progress report of research proposals that investigate different dimensions of communication in a variety of different fields, as a contribution to the design and implementation of integrated communication actions in conversational social processes. The project "Communicative indicators in multiple dimensions" has the goal to construct indicators in four dimensions of communication: informational, interactional, ideological and sociocultural. In this sense, the paper presents tools for construction, selection, survey and registration on the interactional dimension indicators in the environment of a digital platform. This allows a continuous monitoring of activities, processes and products in the context of communication projects and programs within organizations and companies.

\section{Keywords}

Strategic communication, methodology, interactional dimension, communication indicators. 


\section{Introducción}

El proyecto de investigación PID Comunicación estratégica: indicadores de comunicación en dimensiones múltiples. IPOL 172, radicado en la maestría en Comunicación Estratégica de la Universidad Nacional de Rosario, Argentina, se propone investigar la dimensión comunicacional en organizaciones de distintos ámbitos, desde la metaperspectiva teórica y metodológica de la comunicación estratégica ${ }^{1}$ (Massoni, 2003 y 2013), como un aporte al diseño y la implementación de acciones integradas a procesos de cambio social conversacional.

Desde perspectivas clásicas, la medición de la comunicación en las organizaciones y proyectos se ha realizado mediante el registro de indicadores de proceso y de producto ${ }^{2}$. En otros arregistro de indicadores de proceso y de producto ${ }^{2}$. En otros ar-
tículos y espacios del PID, hemos desarrollado aportes en torno tículos y espacios del PID, hemos desarrollado aportes en torno
al debate respecto del estado del arte y los antecedentes, en relación con el desarrollo y definición de indicadores en proyectos de comunicación.

La propuesta consiste en contribuir a la generación de instrumentos metodológicos que desde la comunicación estratégica permitan valorar analíticamente el estado, avances y desarropermitan valorar analíticamente el estado, avances y desarro-
llo de los procesos comunicacionales, considerando la fluidez y la complejidad del fenómeno en su multidimensionalidad. En la complejidad del fenómeno en su multidimensionalidad. En tal sentido, en el proyecto, se desarrollan herramientas para
la construcción, selección, registro y relevamiento de indicadores en distintas dimensiones comunicacionales (informativa, ideológica, interaccional y comunicacional) que permiten un monitoreo continuo de actividades, procesos y productos de

1 Este artículo no se ocupa de la metaperspectiva de la comunicación estratégica, teoría de la comunicación estratégica constituye una innovación respecto del objeto de estudio tradicionalmente abordado en las teorias clásicas de la comunicación social al proponer como tesis central que ta comanlicación es encuentro en la Para quienes estén interesados en aspectos teóricos o metodológicos, desarrollo de técnicas y herramientas y las correspondientes discusiones bibliográficas 2007, 2011 2013 2015. hros y aportes de esta teoria, ver entre otros Massoni 2007, 2011, 2013, 2015. hrps.//com

2 Al respecto de este tipo de medición tradicional, se pueden consultar, entre
otros documentos, indicadores de desarrollo mediático Oficina de la UNESCO Montevideo http://www.unesco.org/new/es/office-in-montevideo/comunicacion - comunicación, en el marco de estrategias comunicacionales en ámbitos diversos: programas, proyectos, organizaciones y empresas.

El objetivo de este artículo es dar cuenta del desarrollo, los alcances y el grado de avance en la construcción de indicadores comunicacionales en la dimensión interaccional. Para ello, se desarrolló una tipología de indicadores comunicacionales, sus correspondientes definiciones y las variables a ser registradas en cada caso. Es parte de reconocer que los indicadores son instrumentos analíticos que proporcionan medidas -cuantitativas y cualitativas- factibles de transformarse en escalas comunes, y cualitativas- factibles de transformarse en escalas comunes, con la finalidad de facilitar su agregación y comparación. Cum-
plen con la función de dar a conocer en forma sintética aquellas plen con la función de dar a conocer en forma sintética aquellas
situaciones que intencionalmente hemos decidido observar, y por lo tanto, proporcionan información sobre componentes específicos de esta, que pueden ser objeto de algún tipo de medición y valoración de las transformaciones operadas (Cisneros, 2009).

El proyecto establece indicadores como "algoritmos fluidos" (Massoni, 2012, p. 12), capaces de acompañar procesos de despliegue de lo comunicacional en dimensiones múltiples ${ }^{4}$. Mediante el desarrollo de una plataforma digital ${ }^{5}$ online, se genera un mapa dinámico de las reconfiguraciones, en torno a la situación que se aborda, para acompañar el despliegue de ensión interaccional, el desarrollo de indicadores incluyó un proceso de validación y la selección de los datos más sensibles a ser procesados para la presentación de posibles tipos de informes para dar cuenta de la dimensión bajo análisis.

"Desplegamos a la comunicación como algoritmo fluido de la diversidad: el modelo y finito de operaciones como método y notación de distintas formas del cálculo en torno a lo comunicacional, entendido como encuentro en la diversidad" (Massoni, 2011). Así, "las Estrategias de comunicación son dispositivos de conversación microvan desplegando en los territorios a partir de las metodologías y técnicas propias de la comunicación estratégica”" (Massoni, 2012)

4 Para multidimensionalidad de la comunicación ver: Massoni, 2003.

5 En Massoni.; Hebe, S.; Menarbino, B. \& Piola, M. "Plataforma online para el registro y operacion de estrategias comunicacionales". En Question, Revista especializada en Periodismo y Comunicación. Vol 1, No 41 Verano (Enero Marzo), 2014, se describen los

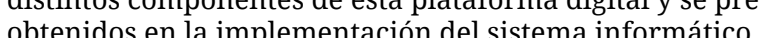




\section{Metodología}

puesta que supera las tradicionales caracterizaciones de receptores y formatos de comuniEl PID trabaja con el modelo de comunicación cación y aún la valorización del conocimiento estratégica de la Escuela de Rosario, y parte de científico como único punto de vista en la comprensión de los fenómenos. Mediante el diseconsiderar a lacomuncación el disemetodologia de investigación enactiva que fa- ño de estrategias comunicacionales, aporta cilita la integración de los saberes científicos a transformaciones en torno a problemáticas en las acciones de desarrollo e innovación en las los territorios, abordando a la comunicación organizaciones, integrando a los propios acto- como espacio de encuentro de la diversidad y res en la producción del conocimiento y en las desarrollando capacidades de los propios actoactividades del proyecto. Se trata de una pro- res como agentes del cambio.

Este proyecto de investigación desarrolla instrumentos metodológicos que abordan a las prácticas como procesos comunicacionales no escindidos, a través de la construcción y el registro de algoritmos fluidos capaces de dar cuenta de las transformaciones en los territorios. Se trata de tion de un sistema articu de instrun específicos de relevamiento y operación para cada una de las dimensiones de la comunicación, desarrollados en procesos micro/macrosociales participativos de apropiación compartida en el entorno de las estrategias de comunicación como dispositivos de investigación enactiva.

\section{Implementación y desarrollo}

El Proyecto de investigación PID Comunicación estratégica: indicadores de comunicación en dimensiones múltiples tiene una duración prevista de cinco años. Dadas sus características, se plantean distintas fases para el desarrollo de indicadores en cada dimensión de la comunicación bajo análisis: dimensión informativa, dimensión interaccional, dimensión ideológica y dimensión del encuentro sociocultural. Cada fase abarca y supera la anterior, aporta productos específicos que no son considerados como finales, en tanto no se completen todos los pasos previstos en la investigación.

Hasta el momento, las etapas desarrolladas en la dimensión interaccional incluyen:

1. Definición de indicadores de comunicación.

2. Validación de la pertinencia, utilidad y relevancia de cada indicador.

3. Maquetación de la plataforma online.

4. Construcción técnica de la matriz de datos: instrumentación de los indicadores seleccionados con los recursos digitales disponibles.

5. Definición de tipos de informes para cada dimensión de la comunicación.
6. Desarrollo de un manual y guía de uso de la aplicación. (En proceso de elaboración)

7. Realización de pruebas piloto: operacionalización de indicadores comunicacionales en distintos proyectos caso. Análisis de resultados. (En proceso de elaboración)

8. Articulación de indicadores en el sistema. (En proceso de elaboración)

En el marco de este proyecto de investigación, fueron publicados una serie de artículos científicos que dan a conocer otros

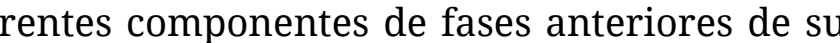
desarrollo. Estos están disponibles en el blogspot del PID: http:// indicadoresdecomunicacion.blogspot.com.ar/

\section{Indicadores interaccionales}

En la dimensión interaccional, se definió una tipología de indicadores comunicacionales, en la cual se enlistaron y caracterizaron los eventos definidos como espacios y momentos de interacción previamente planificados que podrían desarrollarse en diferentes ámbitos organizacionales. Cada categoría de la tipología se estableció a partir de sus rasgos generales, respecto del tipo de encuentro que la sustenta: propósitos comunicacionales, recursos utilizados y modalidades del vínculo intersubjetivo que se establecen en cada estrategia comunicacional como proyecto de investigación enactiva.

Se definieron veinte categorías que fueron validadas por profesionales de la comunicación con perfiles diversos provenientes de los siguientes ámbitos organizacionales: público, privado y de organizaciones no gubernamentales. A través de un formulario de consulta online, y con preguntas abiertas, se consultó sobre el acuerdo o la modificación de las definiciones de cada categoría, sobre la posible superposición de estas y la identificación de otras modalidades de interacción que no hubiesen sido consideradas.

En este proceso, fueron consultados doce informantes clave hasta lograr el punto de saturación de las respuestas. Sus aportes permitieron ajustar la tipología de indicadores en la dimensión interaccional. 


\section{Variables}

Es preciso mencionar que esta tipología de in dicadores se aplica siempre en el entorno de despliegue de una Estrategia Comunicaciona y por ende, en vinculación con una Versión Técnica Comunicacional (VTC). Se definieron un total de veintiséis variables, con el objetivo de cuantificar los espacios de interaccion, vo de cuantificar los espacios de interacción, señalar su vínculo con el componente de correspondiente aspecto del problema. (Por ejemplo, número de participantes, número de oradores (Ver "Variables", más adelante). Así mismo, permite situar los diferentes momentos de interacción y ponderarlos como parte del proceso comunicacional global $c$ lizado.

\section{Procesamiento de la información}

La tipología de indicadores comunicacionales de la dimensión interaccional y sus correspondientes variables forman parte de la estructura de carga de datos de la plataforma online.

Figura 1.

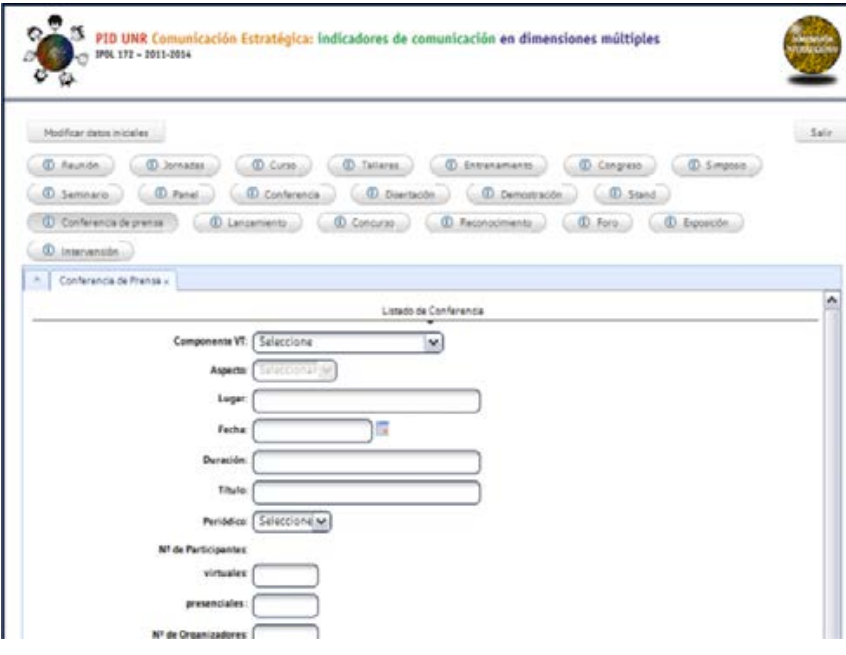

Fuente: Captura de pantalla (www.algoritmosfluidos.com.ar)
Una vez cargados los datos de la tipología de indicadores de la dimensión interaccional de 政 el sistema, se elaboran informes de resultados. can la informán con distintas forn yrafcan la información con distintas formas de visualización de los datos (tablas, barras, tortas, radiales, líneas, nubes, áreas, burbujas, entre otras), según las correlaciones de variables que se busquen explorar en el entorno de cada estrategia comunicacional en particular.

En relación con el procesamiento de la información de la dimensión interaccional, los informes están orientados a dar cuenta de la correlación de procesos y productos comunicacionales en los distintos espacios (cursos, congresos, seminarios, redes sociales). Presentamos ejemplos de estos cruces de información en más adelnate en "Procesamiento de la información”.

\section{Resultados}

En función de la investigación realizada en la dimensión interaccional, se obtuvieron los siguientes productos: tipología de indicadores interaccionales, sus correspondientes variables y posibles informes.

\section{Tipología de indicadores de la} dimensión interaccional

1. Reunión: es un encuentro presencial o virtual con objetivos comunicacionales predefinidos y establecidos en el programa de la convocatoria o surgidos del intercambio realizado. Puede ser una reunión informativa, de planificación, de sistematización, etcétera.

2. Jornada: evento en torno a una problemática Se diferencia de otros por su carácter amplio, heterogéneo y no necesariamente académico ni periódico. Pueden contener propuestas de diversa índole: presentación de novedades técnico-científicas, productos y tecnologías, debates, interacción de actores, consultas con especialistas, prácticas o recorridas demostrativas, espacios de preguntas y respuestas. Pueden estar dirigidas a un público específico o ser abiertas.

3. Curso: espacio de formación compuesto por clases, módulos y niveles, dictado por especialistas y destinado a un público específico, con periodicidad variable. Puede incluir la entrega de una evaluación o trabajo práctico. Por lo general, tiene un objetivo formativo predeterminado. Es posible que incorpore otros eventos tales como visitas guiadas, demostración, panel, entre otras.

4. Taller: espacio de aprendizaje especialmente organizado para la construcción colectiva de conocimiento. Se caracteriza por una form de trabajo que incluye necesariamente la problematización, reflexión y experimentación, a partir de los saberes de los participantes en la situación que se aborda. Supone la panticipación de uno o varios coordinadores del proceso.

5. Entrenamiento: espacio organizado para que los participantes logren replicar un procedimiento. Supone intencionalidad de instrucmiento. Supone intencionalicad de instrucción y repetición precisa, por sobre cion y repen de un nuevo conocimiento.

6. Congreso: encuentro de integrantes de un campode conocimiento científico queselleva a cabo en forma periódica para debatir, intercambiar conocimientos, experiencias y establecer vínculos entre pares. Incluye distintas modalidades: paneles, posters, mesas redondas, conferencias, etcétera.
7. Simposio: encuentro académico o científico de especialistas para desarrollar y examinar un tema en forma detallada desde diversos ángulos, a través de intervenciones individuales, sintéticas y de sucesión continuada. Hay un tiempo de exposición para los expertos, con la posibilidad de que un coordinador resuma las ideas principales y el auditorio formule preguntas.

8. Seminario: encuentro de especialistas alrededor de una temática previamente definida, que interesa trabajar en un ambiente de mutua colaboración. Los participantes co-construyen conocimiento, mediante una secuencia inicial de presentación de ponencias acerca de la temática, enriquecida por el debate e intercambio de los presentes. Suele incluir los roles de coordinación -gerencia el equipo y diseña el dispositivo-, facilitador -encauza la dinámica de interacción-, y en algunos casos relatores -que sistematizan el trabajo de los participantes.

Panel: reunión donde los expositores conversan sobre un asunto en particular, acordado previamente. Está integrado por varias personas y cada una tiene un tiempo pautado para exponer su punto de vista. Luego de las exposiciones se abre el diálogo entre los panelistas, quienes tratan de desarrollar en la conversación la mayor cantidad de aspectos sobre el tema que los convoca. Incluye un coordinador que presenta a los participantes del panel y administra los tiempos de la conversación. Puede incluir una instancia donde el público realice preguntas a los panelistas.

10. Conferencia: evento donde un especialista expone sobre un tema específico, generalmente en un ámbito científico o académico. Suele incluir un momento para que los 
asistentes formulen preguntas. Las conferencias magistrales son espacios destacados en el ámbito del evento. Pueden funcionar de manera independiente o como parte de un evento mayor.

11. Disertación: instancia en la que un disertante desarrolla un tema. Tiene una modalidad expositiva, con participación del público acotada a la formulacion de pregu en un tiempo de tas en un tiempo determinado. Su carácte , no necesariamente académico o científico.

12. Visita demostrativa: es una actividad que muestra un proceso o producto de manera práctica, dinámica y participativa, enfatizando algún aspecto vinculado a determinado producto o servicio. Se denomina renado producto o servicio. Se denomina recorrida, cuando se propone cubrir distintos puntos geográficos con objetivos predeterminados.

13. Stand: espacio de promoción que en e marco de una feria o exposición se propone aportar a la construcción de la identidad de una organización, proyecto o producto ducto interactuando con los visitantes con objetivos comunicacionales específicos. En su ámbito, se planifican diferentes tipos de actividades: distribución de material de comunicación, juegos, exposición o entrega de productos, disertaciones, concursos, entre otros.

14. Conferencia de prensa: reunión para periodistas, convocados por una o más organizaciones para brindar información con el objetivo de que sea publicada en diferentes medios de comunicación. En general, tiene un orden que se inicia con una exposición, seguido de preguntas pautadas previamente o no.

15. Lanzamiento: es el evento de presentación de una nueva cio. Convoca a la prensa y a públicos específicos. El acto está presidido por uno o más presentadores, autoridades o responsables de la novedad. En general, se entrega material escrito o productos para que los participantes experimenten lo que ha sido presentado.

16. Concurso: competencia entre distintos candidatos que aspiran a un premio o puesto que se otorga a aquel que cumpla con los objetivos y condiciones específicos del certamen, por elección de un jurado, ponderación de antecedentes, sorteo entre finalistas, entre otros.

17. Reconocimiento: evento social donde se reconoce a una persona o grupo por los resultados de su actividad, trayectoria de vida, buen desempeño, algún logro específico o la celebración de una fecha o periodo determinado.

18. Foro: es un encuentro presencial o virtual cuyo principal objetivo es el intercambio entre los participantes sobre un tema. Puede tener un coordinador con capacidades de organización del debate (por ejemplo dar turnos para tomar la palabra o presentar a oradores expertos a un auditorio que luego se integra a la discusión), o ser libre. Pueden tener una duración definida previamente 0 ser abiertos.

19. Exposición: en este evento, un grupo de personas se congrega para exponer, intercambiar o vender productos o servicios. Es un espacio organizado con la intención de aumentar la visibilidad. Cuando el foco está puesto en la venta, se denomina feria.

0. Intervención: es una irrupción artística con un objetivo de impacto comunicacional predefinido, realizada en espacios públicos que rompe con lo cotidiano. Se denomina tambien happening (cuando incluye arte espontáneo o improvisación), performance (cuando está previamente planificado el objetivo artístico) o flash-mob (cuando incluye la captación de multitudes que se congregan para generar un hecho artístico efímero que luego se viraliza en redes sociales).

\section{Variables}

Las variables definidas son:

1. Componente de la VTC

2. Lugar

3. Fecha

4. Duración

5. Título

6. Periódico si/no

7. Nro. de participantes

8. Nro. de organizadores

9. Nro. de oradores

10. Instituciones que participan en la organización (cuáles y cantidad)

11. Instituciones que aportan recursos

12. Gratis o pago

13. Entrega de material. Indicar si hubo lecturas previas.

14. Promoción
15. El diseño incorpora evaluaciones anteriores

16. Está prevista una modalidad de vinculación posterior con los asistentes

17. Hubo registro fotográfico

18. Se entregó certificación, suvenir

19. Hubo trabajos prácticos

20. Se cumplió con los objetivos (autoevaluación)

21. Se hizo un informe posterior

22. Menciones online (Apariciones en google)

23. Hashtag

24. Me gusta

25. Compartido

26. Comentarios

\section{Procesamiento de la información}

La plataforma permite relevar información para todas las variables propuestas en la tipología de indicadores para la dimensión interaccional. Los gráficos sugeridos aquí se presentan solo a manera de ejemplo de algunas de las configuraciones diseñadas para facilitar la visualización en los proyectos caso actualmente en desarrollo. El entrecruzamiento de datos ofrecidos en los informes varía en cada estrategia comunicacional, según su VTC y los procesos comunicacionales que se busquen propiciar en ese proyecto en particular. 
Informe 1: frecuencia y localización de los espacios de interacción por componente de la VTC

Mediante gráficos de barras, este informe permite visualizar la realización de eventos considerando ubicación geográfica, cantidad y fecha por componente de la VTC.

\section{Informe 2: espacios de interacción por proceso} comunicacional

Mediante gráficos de barras, este informe permite visualizar la realización de eventos considerando ubicación geográfica, cantidad y fecha por procesos comunicacionales priorizados (infor mación, interacción, motivación y enacción).

\section{Informe 3: espacios de interacción y procesos} comunicacionales por componente de la VTC

Este informe da cuenta de los procesos comunicacionales priorizados (información, interacción, motivación y enacción) por componente de la VTC.

Figura 2

\section{Eventos y procesos comunicacionales por componente de la VTC}

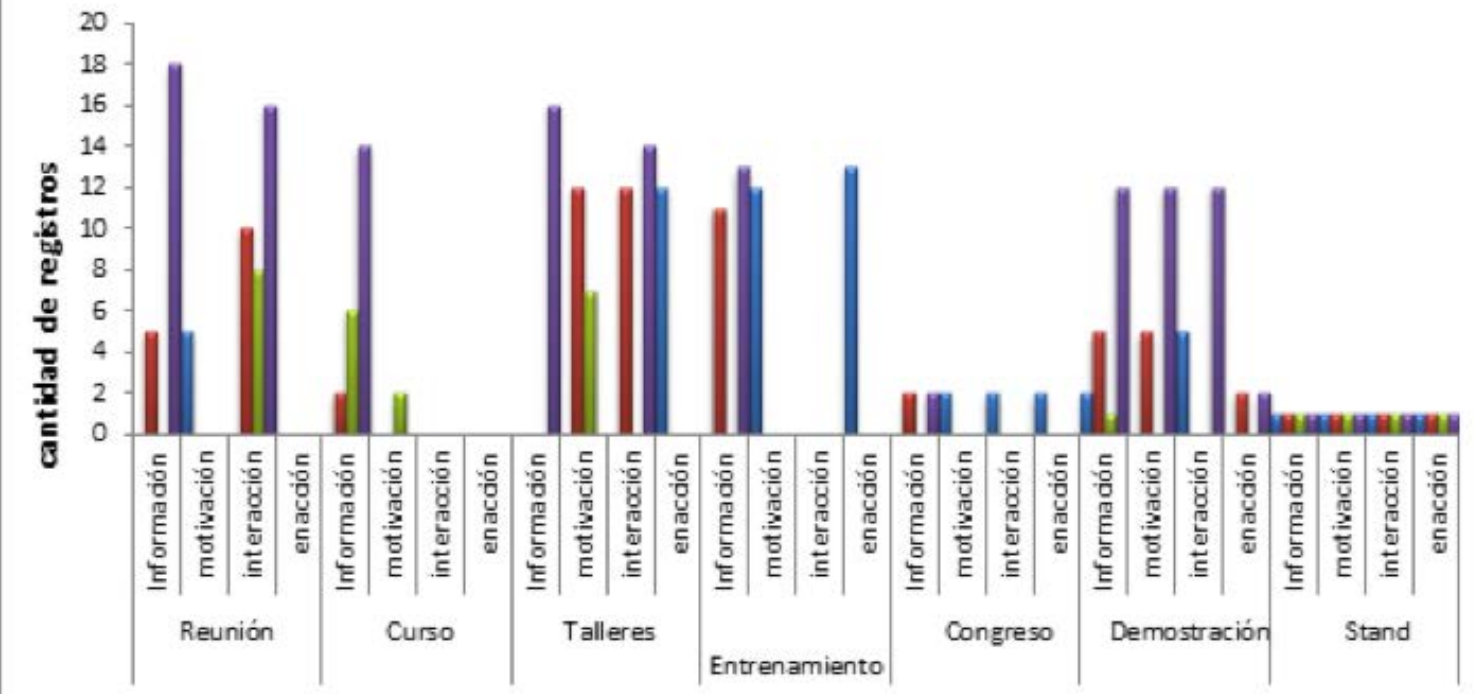

procesos comunicacionales priorizados por evento

\section{Informe 4: espacios de interacción y} organizadores

En este informe, se registra la relación entre tipos de espacios de interacción y organizadores, considerando el tipo y la frecuencia de los recursos aportados.

\section{Informe 5: seguimiento online}

Este informe expresa la relación entre la cantidad de apariciones, menciones y valoraciones en las redes sociales utilizadas en la estrategia comunicacional, según el tipo de espacio de interacción.

\section{Conclusiones}

El proyecto de investigación Comunicación estratégica: indicadores comunicacionales en dimensiones múltiples, donde se inscribe lo desarrollado en este artículo, trabaja en establecer algoritmos fluidos para acompañar el despliegue de lo comunicacional en situación. La definición y operación de indicadores en la dimensión interaccional es un nición y operación de indicadores en la dimensión interaccional es un ción, considerando la complejidad de lo real, rebasando la instancia del resultado, para incorporar indicadores al despliegue de estrategias comunicacionales como procesos fluidos de innovación en los territorios.

Este texto presenta avances y primeros resultados de la investigación, en torno a la dimensión interaccional: definición de la tipología de indicadores (veinte indicadores con sus variables de registro), definición de informes de visualización prediseñados (cinco informes para la dimensión interaccional), validación de los indicadores y ensayo de carga en la plataforma (dos proyectos caso). Esta tipología de indicadores interaccionales, sus correspondientes variables e informes de visualización, son herramientas actualmente integradas a la plataforma online.

Otros aportes de esta fase del proyecto de investigación

- Plataforma digital operativa en la dimensión interaccional que permite ponderar las acciones desarrolladas por componente y por aspecto de la problemática definida en la VTC, en correspondencia con el proceso comunicacional que se busca promover. 
- Tipología de indicadores de la dimensión interaccional que opera como interfaz conversacional hacia el interior del proyecto y a la vez como dispositivo de planificación a la vez como dispositivo de platrategia comunicacional.

- Obtención de Informes en línea que correlacionan productos y procesos comunicacionales de la dimensión interaccional en la situación bajo análisis.

- Registro, seguimiento y monitoreo de la estrategia comunicacional, al mismo tiempo que se está implementando en el territorio.

- Diseño de una interfaz capaz de abordar la multidimensionalidad de la comunicación, incluyendo la dimensión interaccionar.

El sistema propicia el encuentro social conversacional con vistas a lograr una transformación en la dirección deseada en cada fase de la estrategia comunicacional. Las modalidades de visualización de los datos registrados en la plataforma online despliegan derivaciones interesantes. Lejos de presentarse como un complemento de los resultados obtenidos, se comizlemento de los resultados obtenidos, se avizoran como constituyentes articuladores de modalidades innovadoras de intervención en el territorio, a partir de la operación en la dimensión comunicacional. En futuros trabajos, presentaremos los alcances de su implementación en los proyectos caso actualmente bajo estudio.

\section{Nota final}

El equipo de investigadores de proyecto está integrado por Santa Massoni (Directora), Mariana Piola (Co-directora), Mariana Mascotti, riana Piola (Co-directora), Mariana Mascotti, Luciana Margherit, Javier Spagnolo, Bruno Menarvino y Evelyn Gerson. Más información y contacto en http://indicadoresdecomunicacion.blogspot.com.ar/. 而

\section{Referencias}

aa.vv en el sitio de la Escuela de Comunicación Estratégica de Rosario https:// comunicacionestrategicarosario.sites.google.com/site/comunicacionestrategicarosario/

Cisneros, J. (2009). El problema metodológico en la elaboración de indicadores en ciencias sociales. Buenos Aires: Flacso.

Deleuze, G. ¿Qué es un dispositivo? (1990). En Michel Foucault, filósofo; Gedisa; Barcelona.

Massoni, S. (2004). "Estrategias de comunicación: un modelo de abordaje de la dimensión comunicacional para el desarrollo sostenible entendido como cambio social

conversacional”. Resumen de Tesis doctoral en Revista Temas y Debates No 8 , Facultad de Ciencia Política y RRII, Universidad Nacional de Rosario, Rosario, Argentina. UNR Editora, (Con referato)136 p. ISSN 1666- 0714

Massoni, S. (2006). Multi-dimensionality of reality and the communication approach for sustainable development, en Alfonso Gumucio-Dagron and Thomas Tufte (eds.), Communication for social change anthology: historical and contemporary readings, New Jersey, Communication for Social Change Consortium

Massoni, S. (2011). Estrategias. Los desafíos de la comunicación en un mundo fluido. Homo Sapiens Ediciones: Rosario, Argentina.

Massoni, S. (2011). Comunicación Estratégica: comunicación para la innovación. Homo Sapiens Ediciones: Rosario, Argentina.

Massoni, S. Libro CICE, Congreso internacional de Comunicación Estratégica, BUAP, Puebla, 2011. MASSONI, Sandra. Elogio de la comunicación estratégica como algoritmo fluido de la diversidad. Comunicadores a las orillas de otra ciencia y de otra profesión. Estrategias, Revista Académica Año VI No 17, FISEC, Ar. URL de la Revista: www.fisec-estrategias.com. ar. (Con referato). ISSN 1669- 4015, 2012.

Massoni, S. (2013). Metodologías de la Comunicación Estratégica: del inventario al encuentro sociocultural. Homo Sapiens Ediciones: Rosario, Argentina.

Massoni, S.; Mariana, P; Mascotti, M.; Margherit, L.; Spagnolo, J.; Menarvino, B. \& Gerson, E. (2013). Documento PID IPOL 172. Informe de avance de Año 3 (2013). Investigación y posgrado. Facultad de ciencia política y RRII, Universidad Nacional de Rosario: Rosario.

Massoni, S.; Mariana, P; Mascotti, M.; Margherit, L.; Spagnolo, J.; Menarvino, B. \& Gerson, E. (2013). Documento PID IPOL 172. Tipología de indicadores de la dimensión interaccional. Investigación y posgrado. Facultad de ciencia política y RRII, Universidad Nacional de Rosario: Rosario. 
Massoni, S.; Menarbino, B.; Piola, M. (2014). "Plataforma online para el registro y operación de estrategias comunicacionales”. En revista especializada en Periodismo y Comunicación. Vol. 1, Nro. 41 Verano (enero-marzo).

Massoni, S.; Mascotti, M. \& Margherit, L. "Medición de procesos comunicacionales en organizaciones y proyectos: La dimensión informativa desde la comunicación

estratégica”. En revista Latinoamericana Razón y Palabra. Publicación electrónica. Año 17, Nro. 85 diciembre 2013- marzo 2014.

Organización de las Naciones Unidas para la Educación, la Ciencia y la Cultura- Indicadores de Desarrollo Mediático: Marco para evaluar el desarrollo de los medios de comunicación social. Aprobado por el Consejo Intergubernamental del Programa Internacional para el Desarrollo de la Comunicación (PIDC) en su sesión XXVI (26-28 marzo 2008). Referencia no. $\mathrm{CI} / \mathrm{COM} / 2008 / \mathrm{PI} / 3$

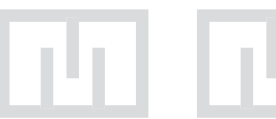

11

11

1

I

\section{Ш} |lit It II II II

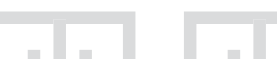

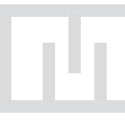

1

II

11

il

1

11

1

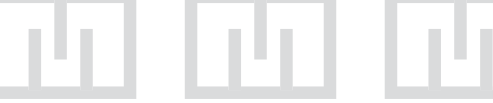

1

प1

1

11

1

11

11

1

11

1

1

1

II

1

D

11

II

1

11

II

1

11

II

1

11

-

1

11

11

11 I I

11

11

11 